SHS Web of Conferences 20, 01002 (2015)

DOI: $10.1051 /$ shsconf/ 20152001002

(C) Owned by the authors, published by EDP Sciences, 2015

\title{
A la conquête de la langue orale et écrite élaborée : entre enjeu méthodologique et apprentissage
}

\section{Conquering the oral and written elaborated language: between methodological challenge and learning}

\author{
Carine Bert ${ }^{1, a}$ \\ ${ }^{1}$ Aix-Marseille Université ADEF EA 4671, 32 rue Eugène Cas, 13248 Marseille Cedex 4, France
}

\begin{abstract}
Résumé. Cet article se fonde sur les différentes étapes de notre recherche sur la langue des élèves, s'inscrivant dans le cadre de la méthodologie expérimentale, de la collecte de données sur le terrain à l'analyse linguistique des productions orales et écrites des élèves. Ce sont les élèves de CM1 (enfants de 9-10 ans) de deux classes du département des Bouches-du-Rhône et de CM2 (enfants de 10-11 ans) d'une classe drômoise qui ont été filmés en situation d'interview de 2013 à 2015. Parallèlement, ils ont réalisé plus de 750 textes manuscrits de différents types. Les enregistrements oraux et les productions écrites ont été retranscrits en corpus et analysés selon les méthodes du Groupe Aixois de Recherche en Syntaxe afin d'en observer les régularités. Cette étude nous a permis de dresser une liste des structures caractéristiques de la langue élaborée et de constater l'apport des différentes situations didactiques sur la construction syntaxique et lexicale des élèves.
\end{abstract}

\begin{abstract}
This paper is based on the various steps of our research on the pupils' language, in the outline of experimental methodology, from collecting school data to analyzing oral and written productions of pupils. Pupils 9-10 years old from two classes in Bouches-du-Rhône department and pupils 10-11 years old from a class in Drôme department were filmed during interview situations from 2013 to 2015 . They realized more than 750 written texts of various types. The oral recordings and written productions were transcribed into corpus and analysed according to the method of the Groupe Aixois de Recherche en Syntaxe in order to observe regularities. This study gave us a list of the characteristic structures of the elaborated language and we could point out what the didactical situations broughtto the syntaxical and lexical constructions of the pupils.
\end{abstract}

\section{Introduction}

Cet article qui s'inscrit dans la continuité du Colloque international ICODOC 2015 intitulé "Corpus complexes et enjeux méthodologiques : de la collecte de données à leur analyse" se propose d'analyser les différentes étapes méthodologiques conduisant à l'analyse de corpus tout en s'appuyant sur nos expérimentations réalisées dans le cadre scolaire avec des élèves de cycle 3 de l'école élémentaire au sein de deux départements : la Drôme et les Bouches-du-Rhône. Ce sont nos recherches, au carrefour de la linguistique française et des sciences de l'éducation, fondées sur l'usage de la langue élaborée, c'est-à-dire d'une langue utilisant des structures syntaxiques complexes et un lexique technique approprié à chaque domaine. Le choix de mettre en place certaines situations didactiques orales filmées nous ont conduits à nous confronter au terrain, à franchir divers obstacles pour parvenir à collecter les données qui allaient constituer la base de notre étude. Afin de permettre au lecteur de saisir notre démarche, nous inscrirons notre problématique dans son contexte social et scientifique, préciserons l'objectif de notre recherche, la méthodologie qui nous a permis de cheminer de la collecte de données à l'analyse pour ensuite proposer quelques résultats et ouvrir sur de nouvelles pistes d'explorations.

\footnotetext{
a Auteur de correspondance : carine.bert@wanadoo.fr
}

This is an Open Access article distributed under the terms of the Creative Commons Attribution License 4.0, which permits unrestricted use, distribution, and reproduction in any medium, provided the original work is properly cited. 
Si notre objet de recherche, l'usage de la langue élaborée par les élèves, a dicté la méthodologie que nous allions employer, c'est que celui-ci était fortement ancré sur le terrain de l'école tout en prenant en compte l'apport de la recherche scientifique. Ainsi, parvenir à apprivoiser le terrain était pour nous la première étape à franchir si nous souhaitions avoir accès à la langue des élèves qui seule pouvait nous fournir les indices de langue élaborée que nous désirions analyser. Commençons donc par définir ce que nous prenons pour référence quand nous parlons de «langue élaborée ». En faisant un tour d'horizon des chercheurs qui se sont intéressés à cette notion, nous trouvons la linguiste Claire Blanche-Benveniste qui définit la langue élaborée comme la «langue du dimanche» (BlancheBenveniste in Roubaud, 2013 [1]) à l'image de l'habit du dimanche réservé aux grandes occasions. Quant à Basil Bernstein (1975 [2]), il opposait déjà dans les années 1970, le code restreint et le code élaboré. Portons désormais notre regard sur l'école, cette langue élaborée y est-elle présente, l'enseigne-t-on ? S'il s'avère qu'elle fait une brève apparition dans les Instructions Officielles en lien avec les registres de langue, elle est le plus souvent absente des progressions des enseignants. Lorsque nous les questionnons à ce sujet, ils justifient son absence dans les enseignements en nous expliquant qu'elle n'est pas évaluée. Afin de mieux comprendre les enjeux de la langue des élèves, nous nous sommes donc référés aux travaux de Marie-Noëlle Roubaud et Paul Cappeau (2005 [3]) sur l'analyse linguistique des textes d'élèves ainsi que sur les situations d'apprentissage relatives au langage, proposées grâce à une collaboration entre enseignants et chercheurs par Emmanuelle Canut (2006 [4]).

En plus d'un enjeu scientifique, nous avons vite constaté l'enjeu social que représentait cette étude relative à l'apprentissage de la langue française à l'école puisque, suite à l'analyse linguistique et en fonction des résultats obtenus, il nous serait désormais possible de concevoir différentes situations didactiques en fonction des structures linguistiques à acquérir. Or, nous avons déjà pu observer à ce jour, l'enrichissement du lexique des élèves et la construction de structures complexes de la langue grâce à la mise en place de situations de parodies, d'interviews en les associant à différents types d'écrits. Ainsi, cela témoigne de l'importance de proposer parallèlement des situations de langage oral et de langage écrit si nous voulons varier les structures linguistiques employées. Face à une école qui privilégie le plus souvent l'écrit au détriment de l'oral, Claire Blanche-Benveniste s'exprimait ainsi : «Opposer la langue parlée à la langue écrite a longtemps été, pour le grand public, une affaire de combat entre le bien et le mal : langue parlée spontanée, éventuellement pittoresque, mais à coup sûr fautive ; langue écrite policée, témoignant, surtout grâce à l'orthographe, de la vraie grammaire de la langue. » (Blanche-Benveniste, 1997 [5]). Dans la continuité des chercheurs (Grandaty \& Turco, 2001[6]) insistant sur les enjeux des discours oraux en classe, nous étions convaincus de l'importance que pourrait revêtir le langage oral dans l'apprentissage du français et nous souhaitions tester différents dispositifs. Les travaux de Claire Blanche-Benveniste (2003 [7]) sur le langage oral soulignaient d'ailleurs l'apport des situations de parodies dans l'apprentissage du français. Dans la même direction, Claude Mayor (1991 [8]) insistait sur l'importance des interactions verbales simulant des personnages.

\section{Analyse}

Notre choix a donc été de mettre en place ces situations de parodies et d'interviews avec des élèves de CM2 (enfants de 10-11 ans) puis de CM1 (enfants de 9-10 ans). Nous avons ainsi pu constater à notre tour l'apport de ce type de situations langagières sur les apprentissages syntaxiques et lexicaux. Les élèves employaient un lexique technique en lien avec le sujet abordé, ils construisaient leurs énoncés en utilisant les formules de politesse, rétablissaient le "ne" de négation, utilisaient de nombreuses subordonnées introduite par "dont", "lorsque"... Cependant, cela n'a pas été immédiat car nous avons vite pris conscience que nombre d'élèves n'avaient jamais entendu certaines structures syntaxiques. C'est ainsi que les propos de Claire Blanche-Benveniste ont pris tout leur sens et que nous avons choisi d'exploiter les modèles dont elle conseillait l'usage : " Pourquoi l'école a-t-elle des difficultés à imaginer un enseignement de langue orale ? L'école a interprété langue orale uniquement dans le sens de langue spontanée. (...) Mais il est important par-dessus tout de montrer des modèles d'une langue de prestige. On n'en montre habituellement que pour l'écrit [...] et les maîtres eux- 
mêmes ont souvent du mal à se présenter comme des détenteurs d'un oral de prestige. » (BlancheBenveniste, 2003 [7]). Nous avons donc proposé aux élèves l'écoute d'interviews de personnalités et notamment de linguistes afin de leur permettre de s'approprier le modèle de l'interview ainsi que le lexique correspondant. Nous avons ainsi pu constater que les enfants retenaient des mots relatifs au langage élaboré de la linguistique comme « corpus », " morphème » ou encore " colloque ».

En voici un exemple extrait de l'interview d'une linguiste par les élèves de CM1 : "nous vous avons entendu à votre interview à la radio et vous parliez de corpus + pouvez-vous nous expliquer ce que c'est"

Comme l'expliquait Claire Blanche-Benveniste, il existe un lien entre choix linguistique et usages syntaxiques-lexicaux du milieu d'origine et si nous voulons rendre accessible à tous la langue élaborée, il est donc nécessaire d'offrir aux élèves l'environnement favorable à son usage. Dans la même direction, Gérard De Vecchi (2010 [9]) insiste sur l'importance du contexte et Françoise Gadet 1989 [10]) associe contexte et variations.

Un autre aspect est à prendre en compte concernant l'école, il s'agit des Instructions Officielles. Si le langage oral et écrit apparaissent dissociés dans les programmes de l'école élémentaire, ils y sont cependant présents tous deux. Par contre, sur les manuels scolaires, l'apprentissage de l'oral est souvent peu présent ou calqué sur l'écrit. De plus, en écoutant les élèves parler, nous notons que la langue familière ou courante sont largement privilégiées. Quelle méthodologie allait donc être la plus propice à favoriser l'emploi de la langue élaborée à l'école ? Puisque les enfants ont très peu d'occasion de faire usage de ce registre de langue, notre objectif a donc été de mettre en place différentes situations telles que la parodie ou l'interview afin d'en observer l'impact sur les acquisitions linguistiques, en faisant l'hypothèse que ces situations susciteraient une construction plus structurée de la langue. Si Claire Blanche Benveniste montrait l'apport de la parodie dans les acquisitions langagières à l'école maternelle (Roubaud, 2013 [1]), nous avons à notre tour constaté, grâce à nos expérimentations, l'apport de la parodie mais aussi des situations d'interviews dans les acquisitions syntaxiques et lexicales des élèves de cycle 3. Cela a été rendu possible grâce au concours de deux classes, au sein de deux écoles de milieu populaire à la riche diversité culturelle. Il s'agissait tout d'abord d'un échantillon de 30 élèves de CM2 puis d'un échantillon de 22 élèves de CM1.

Tout chercheur anticipe certaines problématiques qu'il est susceptible de rencontrer, telles que le fait de trouver un lieu d'expérimentation adapté qui lui fournira les données sur lesquelles se construira son analyse. Nous savions que pour mener à bien cette expérience, il nous fallait organiser nos activités didactiques sous une forme moins traditionnelle et notamment utiliser la vidéo. Puisque l'école est un terrain aux multiples règlementations, des autorisations préalables à toutes expérimentations étaient nécessaires. La démarche est par conséquent toujours identique, il s'agit d'obtenir l'accord du directeur de l'école puis de l'Inspecteur de l'Education Nationale de la circonscription pour lequel nous devons constituer un projet détaillé précisant les objectifs en lien avec les instructions officielles et les programmes. Si nous faisons intervenir le film, il nous faut également obtenir des parents d'élèves, la signature du formulaire d'autorisation de droit à l'image et pour les textes manuscrits, l'autorisation de diffusion des textes d'enfants afin de pouvoir utiliser les supports filmés et partager les supports textuels avec la communauté scientifique. Nous avions pu constater combien cette étape des autorisations était difficile au sein des écoles, les parents nous donnant le plus souvent un accord de principe sans prendre le temps de signer les autorisations. Lors de notre première expérimentation, nous n'étions d'ailleurs jamais parvenus à obtenir l'ensemble des autorisations écrites des parents d'élèves, ce qui ne nous avait pas permis d'utiliser librement les films réalisés.

A cela s'était ajouté le fait que, bien qu'ayant établi une relation de confiance, les enseignants nous refusaient toute forme d'entretien enregistré avec eux, mettant en péril la fiabilité des enquêtes que nous pouvions prendre en note. Nos difficultés n'étaient cependant pas des cas isolés dans le milieu scolaire car Henri Louis Go (2007 [11]) expliquait lui aussi, combien sa démarche avait été semée d'embûches lorsqu'il avait décidé d'observer l'école Freinet de Vence. Le chercheur, souhaitant utiliser une démarche expérimentale, se trouve en effet vite confronté à des enseignants réticents à l'idée d'accueillir un chercheur dans la classe. Comme l'explique Emmanuelle Canut (2006 [4]), la collaboration n'est pas automatique et génère très souvent des tensions. Dans certains contextes, 
l'expérimentation s'en trouve facilitée par une collaboration active d'enseignants désireux de comprendre la langue de leurs élèves, comme ce fut le cas, lorsqu'à la demande de la ville de Romans dans la Drôme, une recherche-action sur « le français parlé d'enfants de migrants en grande section de maternelle » fut confiée à Claire Blanche-Benveniste (Roubaud, 2013 [1]). Si la demande peut provenir parfois du terrain, l'initiative en revient souvent au chercheur qui peut faciliter son entrée dans l'école en inscrivant sa recherche au sein d'un projet de classe et en apportant un complément d'enseignement dans un domaine non pratiqué par le professeur. C'est cette configuration que nous avions connue en choisissant d'expérimenter l'apport de la parodie dans l'apprentissage de la langue française à l'école élémentaire avec une classe de CM2 de la Drôme (Bert \& Roubaud, 2014 [12]). Le directeur nous avait en effet confié sa classe chaque semaine durant six mois en échange de la réalisation d'un projet art, sciences et langage. Cependant, nous avions à faire face à la complexité des conditions de tournage, profitant de chaque recoin de l'école pour réaliser nos films en fonction de la disponibilité des lieux, souvent interrompus dans nos enregistrements, utilisant notre propre matériel vidéo tout en assurant la gestion du groupe d'élèves qui nous était confié. De plus, nous n'avions la classe que ponctuellement, parfois sur le temps de classe, parfois sur le temps périscolaire, ce qui ne permettait pas de construire un projet dans la continuité. A cela s'ajoutait le fait que, nos rencontres avec les élèves étaient souvent espacées, que nous devions nous adapter aux activités de l'école. Une idée nous effleurait souvent l'esprit, celle d'avoir notre propre classe, de pouvoir effectuer nos recherches avec nos élèves.

Face à cette situation et pensant que la solution idéale serait de cumuler les deux fonctions de chercheur et de professeur des écoles, nous avons eu le titre de professeur des écoles et avons mis en place en 2014-2015 une nouvelle observation sur le terrain en offrant aux élèves de notre classe de CM1, l'opportunité d'interviewer une linguiste. Nous pensions que notre recherche en serait facilitée et qu'associer réflexion didactique et pratique de terrain pourrait être fort bénéfique, tout cela sans imaginer les obstacles qui nous attendaient. S'il fut facile d'établir un rapport de confiance avec nos élèves et la directrice de l'école, cela fut source de conflits de la part de collègues qui considéraient ces pratiques comme un jeu non productif sans y avoir assisté. Si nous avons choisi de garder le cap et de poursuivre nos expérimentations dans ce contexte difficile, c'est qu'il nous offrait la possibilité de varier les situations langagières, que les élèves progressaient vite en maîtrise de la langue française, qu'ils s'investissaient totalement dans ce genre de pratique et surtout que l'Inspectrice de la circonscription semblait quant à elle y être favorable.

Ces expérimentations ont enrichi notre corpus de plusieurs heures d'enregistrements vidéo et de nombreux textes manuscrits d'élèves de CM1 et CM2, affinant ainsi notre banque de données relative à la langue des élèves. Ces écrits font intervenir différents types de textes comme le descriptif, l'argumentatif, le dialogue, le narratif, l'informatif ou encore le prescriptif. Ce choix de varier les supports d'écriture nous permet d'obtenir des structures syntaxiques et lexicales diversifiées. Sur l'ensemble des textes, nous avons constaté que le descriptif et l'argumentatif arrivaient en première position à l'écrit alors qu'à l'oral le dialogue et le narratif se plaçaient au premier plan. Cela est bien entendu en lien direct avec le niveau de classe dans lequel les textes sont réalisés, les progressions relatives au Bulletin Officiel de la classe de CM1 insistant sur l'apprentissage du dialogue et de la description.

Voici comment se répartissent les productions écrites et orales par types de texte : 


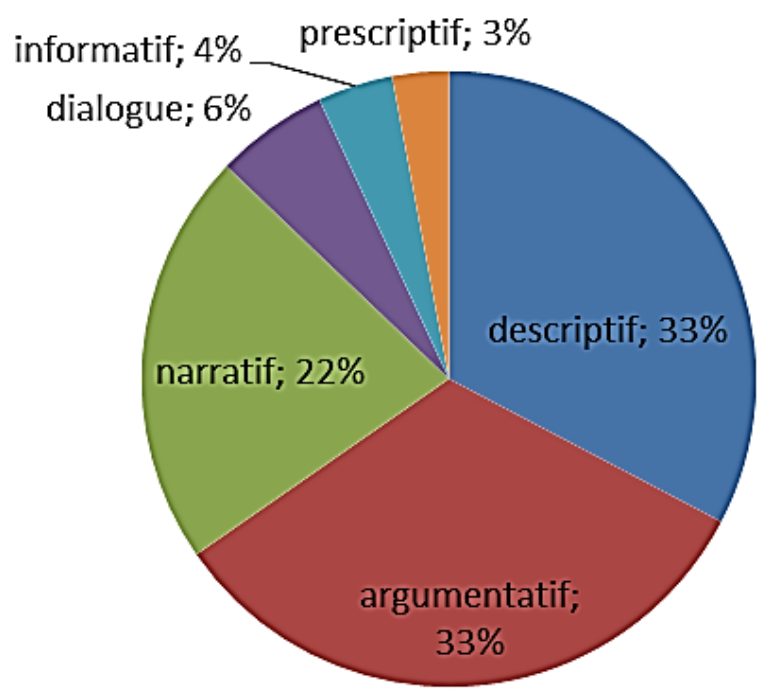

Graphique 1. Nombre total de textes manuscrits (755).

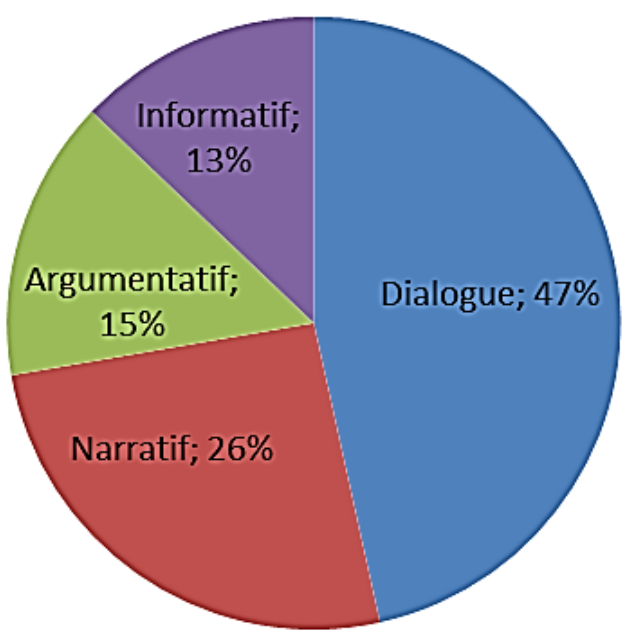

Graphique 2. Durée totale des enregistrements oraux (6h $23 \mathrm{~min}$ ).

La présentation sous forme de diagramme en secteurs nous indique clairement la répartition des types de textes et nous permet d'observer que l'association des situations de langage oral et écrit favorise la diversité des usages de la langue.

Face à la collecte de données multi-modales qui s'amplifiait, nous avons vite été confrontés aux enjeux méthodologiques de la constitution du corpus et à l'importance du codage dans la transcription informatisée. Pour la transcription de la langue orale, nous avons choisi d'utiliser les codes du Groupe Aixois de Recherche en Syntaxe (GARS) (Blanche-Benveniste, 2005 [13]) et conservons les séquences audio ou vidéo d'origine, le film constitué et le corpus retranscrit. Puisque l'enregistrement a un impact direct sur le recueil de données et la transcription, nous utilisons pour nos enregistrements, un appareil photo numérique de qualité ou un dictaphone et rassemblons les données en utilisant les logiciels de montage Studio 18 pour la vidéo ou Audacity pour le son.

Voici un exemple de transcription d'un énoncé oral : 
L : nous sommes heureux de vous avoir parmi nous + merci d'avoir répondu à notre invitation + notre maîtresse nous a beaucoup parlé de vous cependant nous aimerions en savoir davantage + j'aurais quelques questions à vous poser + pourquoi avez-vous choisi le métier de linguiste

Puisqu'il s'agit de discours oral, nous n'indiquons aucune ponctuation ni majuscules dans la transcription, seules les pauses sont précisées.

Pour les textes manuscrits, nous recherchons un codage qui facilitera les recherches lexicales ou syntaxiques, l'écriture non normative des élèves complexifiant la constitution du corpus et nécessitant pour chaque texte plusieurs versions : le manuscrit original, la transcription informatisée conforme au texte d'origine et la version corrigée conservant quelques traces non normatives.

Le corpus constitué, il s'agissait désormais de faire parler les données. Nous nous sommes tout d'abord intéressés aux différentes représentations des élèves sur la langue élaborée en analysant les divers questionnaires remplis par les élèves de trois écoles différentes de la Drôme et des Bouches du Rhône, soit plus de 100 élèves. Pour les élèves, l'écrit prenait une dimension d'apprentissage : « apprendre les mots, la langue » contrairement à l'oral qu'ils considèrent davantage comme un média d'apprentissage «l'oral est pour bien expliquer les exercices». Les réponses apportées nous montrent clairement que pour les élèves, l'écrit est le véritable objet d'apprentissage alors que l'oral est davantage considéré comme un outil de communication au service des apprentissages. Une inégalité apparaît donc entre le statut de l'écrit et celui de l'oral.

Dans un second questionnaire où les élèves de CM1 devaient définir ce qu'était pour eux une langue soutenue, ils utilisèrent des termes qui les distanciaient de cet usage de la langue élaborée, comme si celle-ci leur semblait inaccessible : "ça veut dire une langue comme une reine », "une langue qui est très très polie».

Ensuite, cette intuition nous a été confirmée lorsque nous avons demandé aux élèves qui auraient pu prononcer l'énoncé suivant : «une éolienne est une tour qui sert à produire de l'électricité en utilisant une énergie renouvelable qui est le vent. »

Ils attribuèrent ces propos en grande majorité aux scientifiques et techniciens comme en témoigne notre collecte de données. Aucun élève n'a associé cette formulation à des enfants. Or, elle avait été énoncée par des élèves de CM2 en situation d'interview. Voici la représentation sous forme de diagramme en secteurs qui illustre cette situation :

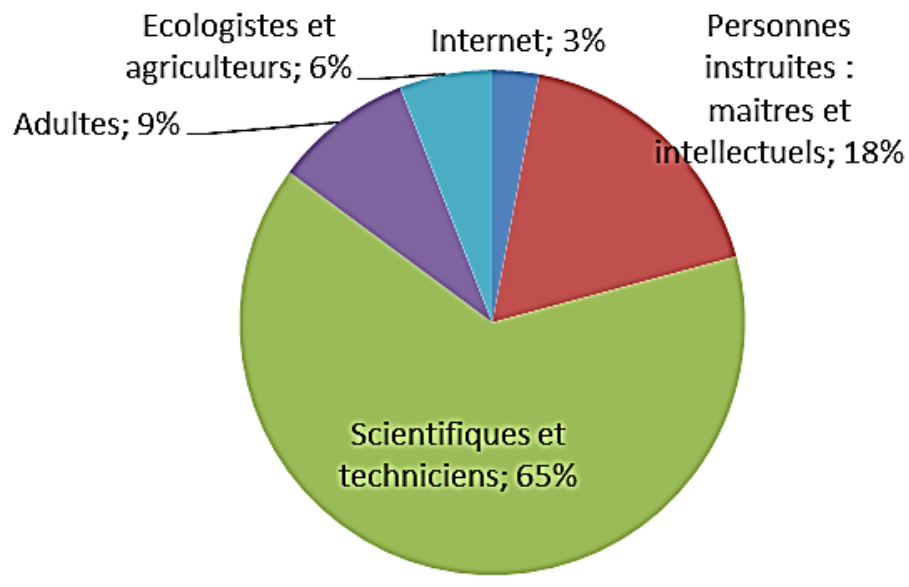

Graphique 3. Personnes utilisant un langage technique recherché.

Puis, nous avons réalisé une étude linguistique approfondie des structures syntaxiques et lexicales des productions orales et écrites des élèves mis en situation d'interviews afin d'en dégager les indices de langage élaboré et d'en dresser une liste. Nous avons également utilisé la technique de la «mise en grilles » (Blanche-Benveniste, 1990 [14]) qui nous a permis de faire ressortir les axes paradigmatiques et syntagmatiques du discours, révélant ainsi des régularités syntaxiques du discours telles que la symétrie ou le parallélisme. Ces grilles nous ont aussi offert la possibilité de distinguer les différentes 
recherches de dénomination, nous donnant ainsi des indications sur la construction du lexique par les élèves.

Voici un exemple de mise en grille d'un énoncé d'un élève de CM2 en situation d'interview où les effets de symétrie et de parallélisme sont bien visibles :

et

quand le sol est perméable quand le sol est imperméable

et quand elle ne peut pas s'infiltrer ces précipitations

elles

elles

elle ruissellent

rejoignent les rivières

s'infiltrent

rejoignent

rejoint les nappes phréatiques

les mers

Voici un exemple de recherche de dénomination d'un élève de CM2 en situation d'interview où l'erreur d'accord donne une indication sur la complexité de construction du discours :

une éolienne est fait

$\begin{array}{llll} & \text { de } & & \\ & \text { d' } & \text { une hélice } & \text { constituée de trois pales } \\ & \text { d' } & \text { un engrenage } & \\ & \text { d' } & \text { un mât } & \\ \text { et } & \text { d' } & \text { un alternat- } & \\ & \text { d' } & \text { un multiplicateur } & \text { qui transforme l'énergie mécanique en électricité }\end{array}$

Si, comme l'explique Emmanuelle Canut (2006 [4]), les structures syntaxiques employées par les enfants sont liées aux habitudes langagières du milieu d'origine, ces situations nouvelles, offrant des modèles de langage élaboré, favoriseraient donc l'usage de structures syntaxiques et lexicales élaborées et par conséquent enrichiraient l'apprentissage de la langue française. Nos recherches nous ont par conséquent permis d'observer combien la problématique du terrain, avec sa réglementation et son environnement, conditionnait la constitution de corpus complexes mais aussi amenait à concevoir des situations nouvelles en les adaptant à chaque contexte scolaire. En procédant ainsi, à l'image de la classe d'André Giordan (1998 [15]), notre classe devient le cadre d'une pièce de théâtre sans cesse renouvelée où le chercheur et professeur explore, tel un metteur en scène, les situations langagières les plus favorables aux acquisitions linguistiques... C'est ce que nous avons entrepris en développant l'interview (Bert, 2015 [16]) au sein de l'école et que nous poursuivons actuellement.

\section{Conclusion}

Pour conclure, nous pourrions dire qu'ayant constaté dans les premiers énoncés et textes des élèves en nous appuyant sur la linguistique de corpus une faible représentation de la langue élaborée telle que nous l'avons définie, notre objectif avait été, dès le départ, d'en comprendre l'origine puis de réfléchir à la manière dont nous pourrions favoriser un usage plus large de cette langue élaborée. Nous souhaitions montrer par nos expérimentations, l'intérêt qu'elle pourrait représenter à s'exprimer davantage à l'école et son impact sur les apprentissages. Ainsi, nous avons choisi d'analyser les structures syntaxiques et lexicales dans différentes situations didactiques, ce qui nous a permis de constater que les situations d'interviews favorisaient particulièrement l'emploi d'un lexique technique recherché et l'usage de constructions riches en subordonnées. De plus, le fait de proposer des modèles en facilitait la mobilisation. Dans la continuité des travaux de Claire Blanche-Benveniste sur l'importance de développer conjointement langue orale et écrite, nous avons ainsi montré comment les élèves, mis en situations de produire un langage élaboré, jouaient avec les mots et les constructions élaborées vers une plus grande maîtrise de la langue française. Notre expérimentation transposant à l'école la démarche du metteur en scène nous ouvre ainsi de nouvelles perspectives et nous encourage à explorer d'autres situations didactiques afin de pouvoir apporter une nouvelle pierre à ce champ que représente l'apprentissage de la langue française à l'école. 


\section{Bibliographie}

1. M.N. Roubaud. Langue et enseignement. Une sélection de 22 manuscrits de Claire BlancheBenveniste (de 1976 à 2008). Tranel, 58. (2013)

2. B. Bernstein, B. Langage et classes sociales. (1975)

3. P. Capeau et MN. Roubaud, Enseigner les outils de la langue avec les productions d'élèves. (2005)

4. E. Canut, Apprentissage du langage oral et accès à l'écrit : Travailler avec un chercheur dans l'école. (2006)

5. C. Blanche-Benveniste, Approche de la langue parlée en français. (1997)

6. M. Grandaty, G. Turco (Coord.) L'oral dans la classe. (2001)

7. C. Blanche-Benveniste, L'oral des adultes parodié par les enfants. L'école Valdôtaine 59, Cahier pédagogique, nouvelle série. (2003)

8. C. Mayor, L'expression orale dans des situations simulées : Les règles du jeu. In M. Wirthner, D. Martin, Ph. Perrenoud. Parole étouffée, parole libérée. Fondements et limites d'une pédagogie de l'oral. (1991)

9. G. De Vechi, Aider les élèves à apprendre. (2010)

10. F. Gadet, Le français ordinaire. (1989)

11. H. L. Go, Freinet à Vence : Vers une reconstruction de la forme scolaire. (2007)

12. C. Bert, M.N. Roubaud, Parler devant une caméra, Les Cahiers Pédagogiques, 512 : 34-35 (2014)

13. C. Blanche-Benveniste, Le corpus de français parlé du GARS. (2005)

14. C. Blanche-Benveniste, Un modèle d'analyse syntaxique 'en grilles' pour les productions orales, pp. 11-28 (1990)

15. Giordan, Apprendre! (1998)

16. Bert, L'interview : un outil propice aux progrès, Les Cahiers Pédagogiques, 522 (2015) 